\title{
Sistematización teórica sobre la competencia investigativa
}

\author{
Theoretical Systematization of Research Competence
}

\author{
Odiel Estrada Molina ${ }^{1}$ \\ Universidad de las Ciencias Informáticas de Cuba \\ La Habana, Cuba \\ oestrada@uci.cu
}

Recibido 24 de octubre de 2013 • Corregido 13 de marzo de 2014 • Aceptado 2 de abril de 2014

\begin{abstract}
Resumen. Es un artículo de revisión bibliográfica donde se evidencia que la formación de competencia en la educación superior ha tomado mayor auge en el siglo XXI, y una de las competencias en las cuales se ha trabajado es la investigativa. Este trabajo tiene como objetivo realizar una sistematización teórica a partir de 1999-2013 acerca de las investigaciones existentes para la formación y desarrollo de la competencia investigativa en la educación superior, partiendo de su concepto, y las relaciones entre cada uno de sus componentes y dimensiones. Para la realización de la investigación se utilizaron como método investigativo el histórico-lógico y el analítico-sintético y como técnica el análisis documental de 40 investigaciones presentadas por autores europeos, asiáticos y americanos (América del Norte y América del Sur). En el trabajo se presenta un análisis pedagógico de los resultados obtenidos en la investigación, se identifican fortalezas y debilidades, y además se proponen una serie de consideraciones pedagógicas a tener en cuenta para la formación y desarrollo de dicha competencia. El principal resultado que se obtuvo es que no se ha logrado integrar completamente un componente profesional y otro tecnológico a la estructura de la competencia investigativa.
\end{abstract}

Palabras claves. Competencia, educación superior, investigación pedagógica, investigación teórica.

1 Profesor e investigador del Departamento de Ciencias Sociales y Humanidades de la Facultad 6 de la Universidad de las Ciencias Informáticas de Cuba. Las líneas de investigación del profesor son: la formación y desarrollo de competencias en la educación superior y la formación del ingeniero en ciencias informáticas desde la relación academia-industria y bajo el sustento de las tecnologías de la información y las comunicaciones (TIC). Es miembro del equipo editorial de la Revista Cubana de Ingeniería, la Revista Avanzada Científica, la Revista Didasc@lia: Didáctica y Educación y de la Revista Educación en Ingeniería. Ha escrito artículos sobre la formación de competencias, la formación de valores y el desarrollo de software. Actualmente es aspirante a Doctor en Ciencias Pedagógicas. Dirección postal institucional: Km 2 $1 \frac{1}{2}$ Autopista La Habana - San Antonio de los Baños, La Habana, Cuba. Página de la universidad http:// www.uci.cu/ Teléfono: (+53) 53-7-837-2555 
doi: http://dx.doi.org/10.15359/ree.18-2.9

URL: http://www.una.ac.cr/educare

CORREO: educare@una.cr

Abstract. This paper constitutes a literature review that evidences that competency acquisition in higher education has boomed in the XXI century and that one of the competencies developed is research. The objective of this paper is to prepare a theoretical systematization of the years 1999-2013 using the existing research projects regarding the acquisition and development of research competence in higher education, based on its concept and the relationships between each of its components and dimensions. The research methods used were historical-logical and analytic-synthetic and the technique used was the document analysis of 40 papers by authors from Europe, Asia and the Americas. The paper presents a pedagogical analysis of the results obtained, identifies strengths and weaknesses, and proposes a number of pedagogical considerations to be included in the acquisition and development of this competence. The main result obtained is that neither a professional nor a technological component has been fully integrated to the research competence structure.

Keywords. Competence, higher education, educational research, theoretical research.

El desarrollo de la competencia investigativa en la educación superior ha sido objeto de debate y análisis a nivel internacional, ya que esta permite a los egresados universitarios brindar solución a problemas profesionales desde una concepción científica e investigativa. Su formación y desarrollo se encuentra condicionado por el currículo de una carrera universitaria que responde a intereses, necesidades y demandas de la sociedad y los recursos personales que posee el individuo; también, como afirman Barrón (2000) e Irigoin y Vargas, (2002) por la Organización para la Cooperación y Desarrollo Económico (OCDE) al estipular la necesidad de formar individuos competentes en la producción de conocimiento, la economía y el nivel de desarrollo tecnológico con el crecimiento de un país.

El desarrollo de la tecnología, la ciencia, la técnica y las necesidades de la sociedad demandan que los nuevos profesionales universitarios puedan dar solución, de forma científica e investigativa, a problemas profesionales desde una óptica interdisciplinaria, intradisciplinaria y transdisciplinaria (Pérez y Setien, 2008). Esta formación analizada desde la pedagogía resulta compleja, ya que todos los individuos poseen una personalidad única e irrepetible, y su forma de asimilación, apropiación y exteriorización de la cultura aprendida es única también.

La necesidad de desarrollar dicha competencia siempre ha existido. Se han implementado disímiles vías que permiten guiar ese camino, tales son los casos de la inclusión de asignaturas en los currículos que permitan al estudiantado apropiarse de conocimientos y habilidades en la metodología de la investigación científica, las clases tipo seminarios y tipo taller, la orientación de tareas investigativas individuales y grupales, y la elaboración de tesis de pregrado y de trabajos investigativos finales en diversas asignaturas.

Todas estas vías permiten, de alguna manera, que el estudiantado pueda ir desarrollando la competencia investigativa en correspondencia con posiciones teóricas, teorías, corrientes o enfoques pedagógicos determinados (depende del constructo teórico que algunos autores asumen): el funcionalista, el conductual, el constructivista y el enfoque complejo (Tobón, 2008b). 
doi: http://dx.doi.org/10.15359/ree.18-2.9

URL: http://www.una.ac.cr/educare

CORREO: educare@una.cr

Ahora bien, se han obtenido diversas alternativas pedagógicas que permiten el desarrollo de esta competencia; pero: ¿Las conceptualizaciones que se han definido acerca de la competencia investigativa contribuyen plenamente a guiar un proceso pedagógico en la formación multilateral y armónica del estudiantado de forma planificada, dirigida e intencionada? ¿Las estructuras conceptuales que se han propuesto permiten el desarrollo de dicha competencia teniendo en cuenta todos los aspectos de la personalidad? ¿Cuán cercano o lejano se encuentran los resultados pedagógicos obtenidos en las investigaciones realizadas en el desarrollo de esta competencia teniendo en cuenta la formación de la personalidad del individuo y otros variables como la tecnología, de lo que se espera por la sociedad en la solución de los problemas profesionales?

Estas preguntas dieron comienzo a esta investigación, en la que se tiene como objetivo realizar una sistematización teórica de las investigaciones llevadas a cabo desde 1999 hasta la actualidad (2013, siglo XXI) elaborados por autores de diferentes continentes, para constatar cómo se ha venido trabajado la formación y desarrollo de la competencia investigativa en función de las preguntas formuladas anteriormente. El análisis pedagógico que se brinda es a partir del enfoque histórico-cultural de Vygotsky (1982) y sus continuadores como L. I. Bozhovich, Subbotsky, Kraftchenko, Leontiev, Beatriz Castellanos, entre otros.

Este trabajo consta de cuatro secciones principales: 1) la introducción donde se expone la necesidad que dio surgimiento a esta investigación; 2) la metodología, donde se expresa los métodos científicos utilizados en la investigación, así como la determinación de los modelos teóricos existentes acerca del concepto de competencia investigativa y las dimensiones que la integran; 3) la discusión donde se presenta el análisis pedagógico realizado acerca de los modelos teóricos existentes, los cuales fueron integrados debido a sus regularidades en cinco grupos; y 4) consideraciones pedagógicas a tener en cuenta para futuras investigaciones acerca de la formación y desarrollo de la competencia investigativa.

\section{Metodología}

Esta investigación se realizó haciendo uso de métodos de investigación educativa, para ello se utilizaron métodos propuestos por (Colectivo de Autores, 2003). Se realizó una sistematización teórica del concepto de competencia investigativa y de la estructura que esta presenta.

Se realizó una análisis documental de los conceptos abordados por varios autores, principalmente de Europa, Asia y América, este último se separó en autores de América del Norte y de América del Sur. Se utilizó el método histórico-lógico y el analítico-sintético con el propósito de constatar cómo se ha abordado el concepto de competencia investigativa desde 1999 hasta la actualidad, 2013, y para determinar la estructura que estos conceptos presentan sobre el término de competencia.

Se consultaron varias tesis doctorales, artículos en revistas académicas, informes de proyectos educativas internacionales, libros y actas de eventos científicos. 
doi: http://dx.doi.org/10.15359/ree.18-2.9

URL: http://www.una.ac.cr/educare

CORREO: educare@una.cr

Al identificar los conceptos asociados a la competencia investigativa y su estructura, se procedió a analizar las dimensiones e indicadores que se proponen para la formación, desarrollo y evaluación de dicha compentencia. Posteriormente se realizó una análisis pedagógico acerca de los conceptos citados por los diversos autores tomando como referente teórico el enfoque histórico-cultural del Vygotsky.

A partir de la realización de una sistematización teórica, se agruparon los autores de acuerdo con las regularidades existentes entre ellos conformándose así cinco grupos, de la siguiente manera:

El grupo 1: Association pour l'emploi des cadres [France] y Deloitte [France], (2010); Arbeláez, Hernández y Pérez (2006); Balbo (2008), Chu, Chow, Tse y Collier (2008); Contreras (2010); Gayol, Montenegro, Tarrés y D'Ottavio (2008); Irigoin y Vargas (2002): Maldonado et al. (2007); Ortega y Jaik (2010), y Rodríguez (2000).

El grupo 2: Baltrūna (2013); Barnett y Muth (2008); Bezrukova y Bezrukov (2013); Castillo (2008, 2011); Muñoz, Quintero, y Munévar (2006); Quigley y Kuhne (1997) y Velásquez (2007).

El grupo 3: Bolgzda y Olehnovica (2012); Burke et al. (2005); Connor (1972); Delamare y Winterton (2005); Fernández, Llivina, Arencibia, Hernández, y Castellanos (2003); Gallardo (2003); Glass, Vessey y Ramesh (2002); Gray (2007); Hakkarainen, Lipponen, Jarvela y Niemivirta (1999); Lee, Miozzoa y Laredob (2010); Pla (2005); Proyecto Tuning-América Latina (2007) y Sánchez y Tejeda (2010).

El grupo 4: Cejas (2006); Gómez (2009) y Santos (2005).

El grupo 5: Morin (2002); Tobón (2008a);Tobón (2008b) y Tobón, Rial, Ángel y García (2006).

\section{Las competencias investigativas en la educación superior}

La formación y desarrollo de la competencia investigativa ha sido concebida, en la educación superior, tanto en el plano procesal-práctico y estructural-formal del currículo a lo largo de la carrera universitaria, aunque tiene su máxima expresión en la realización de tesis de pregrado y trabajos investigativos finales de diversas asignaturas, ya que el estudiante debe evidenciar el uso de la metodología de la investigación científica en solución de un problema investigativo aplicando para ello todas las etapas de la investigación científica.

Para lograr una formación investigativa adecuada en nuestros estudiantes universitarios, y que estos evidencien un desarrollo de la competencia investigativa se hace necesario realizar acciones pedagógicas que tributen a este fin, teniendo en cuenta la formación de la personalidad del individuo. Esta afirmación pudiera parecer algo trivial, pero su respuesta 
doi: http://dx.doi.org/10.15359/ree.18-2.9

URL: http://www.una.ac.cr/educare

CORREO: educare@una.cr

radica en las posiciones o enfoques teóricos en que se sustenta la educación superior de una institución universitaria en cuanto a la formación de competencias.

En los últimos catorce años, se han realizado varias investigaciones asociadas a la formación y desarrollo de la competencia investigativa, debido, entre otros factores, a las exigencias actuales del Siglo XXI, tales como: la intensificación de la relación universidad-industria, la formación de profesionales con una perspectiva transdisciplinaria, los adelantos tecnológicos y la investigación científica en equipos multidisciplinarios donde sus integrantes no se encuentran en un mismo lugar, dígase país o continente, así como la utilización de las tecnologías de la información y las comunicaciones en las investigaciones científicas y tecnológicas.

Se ha venido perfeccionado en estos años la formación de profesionales universitarios que posean un desarrollo adecuado de la competencia investigativa, que le permita resolver problemas profesionales con el uso del método científico e investigativo.

Para la realización de este trabajo se parte de determinados sustentos teóricos. Al aceptar la filosofía dialéctico-materialista y el pensamiento humanista como fundamentos teóricos generales, los fundamentos sociológicos, psicológicos y pedagógicos poseen una adecuada coincidencia con estos enfoques.

Como fundamento sociológico se parte de que la formación y desarrollo de la competencia investigativa tiene un carácter social, ello exige ser interpretado en relación con otras esferas sociales -política, economía, ciencia, cultura y otras- por las que está condicionado.

Como fundamento psicológico se parte del enfoque histórico-cultural, donde se considera la conjunción dinámica de lo cultural, lo psicológico y lo biológico como fuerza motriz del desarrollo psíquico, que surgen de la premisa dialéctico-materialista de que la persona humana es un ser social por naturaleza, un producto de la sociedad, de ahí el origen social de las funciones psíquicas superiores. De este modo, el desarrollo del estudiantado está determinado por la apropiación de los contenidos profesionales, que se producen en condiciones de integración y en un proceso de construcción interactiva entre los componentes personales del proceso de enseñanza-aprendizaje (estudiante-grupo-profesorado, este último actuando como mediador).

El fundamento pedagógico que se asume es en correspondencia con el enfoque histórico-cultural en el que se destacan la premisa de que la educación conduce al desarrollo; la relación cognitivo-afectivo en el proceso de enseñanza-aprendizaje; la relación teoría práctica; la educación a través de la educación, y la relación entre la institución educativa y el contexto social.

A continuación se ofrecen revisiones teóricas del concepto de competencia investigativa en la educación superior, desde 1999-2013, realizándose un análisis pedagógico determinando fortalezas y debilidades. 
doi: http://dx.doi.org/10.15359/ree.18-2.9

URL: http://www.una.ac.cr/educare

CORREO: educare@una.cr

\section{El análisis pedagógico}

\section{Fortalezas y debilidades del grupo 1}

Este primer grupo aborda la competencia investigativa como una integración de dimensiones cognitivas (conocimientos, habilidades), cualidades personales (actitudes, destrezas) y metacognitivas que permiten al estudiante desempeñarse correctamente en un actividad de investigación.

Maldonado et al. (2007) sostuvieron que "la competencia investigativa propende por la aplicación de los conocimientos, enfatizando en las diferentes esferas involucradas en la actividad investigativa entre las que se destacan las dimensiones epistemológica, metodológica, técnica y social" (p. 48). Esta concepción trae consigo que se limita la competencia a la aplicación de conocimientos asociados a la investigación científica, sin tener en cuenta que el saber, no implica el saber ser, ni saber hacer.

Según estos autores, la competencia investigativa se encuentra conformada por tres dimensiones: la cognitiva, la de cualidades personales y la metacognitiva. Su integración contribuye a un desempeño adecuado en la actividad investigativa. Esta concepción evidencia un enfoque integrador / contextualista del individuo, quien para desempeñarse de forma eficiente debe movilizar todos sus recursos personales, integrando sus conocimentos, habilidades y actitudes.

Esta concepción posee como fortaleza, pues tiene en cuenta la participación en grupos, la gestión del conocimiento, el ejercicio del liderazgo, las relaciones interpersonales, la persistencia ante las dificultades, la disposición para el trabajo en equipo, la actitud crítica ante diferentes alternativas de solución y la actitud emprendedora.

Esto trae consigo que desde la actividad pedagógica y didáctica del profesorado se diseñen estrategias que permitan que el estudiantado pueda desempeñarse como investigador a partir del trabajo grupal y la realización de distintos roles, la capacidad para fundamentar criterios personales o del grupo, la utilización del vocabulario técnico, la actitud positiva ante logros y fracasos, entre otros aspectos propios del individuo competente.

En el orden psicológico se comprende la competencia como una integración, por lo que ninguna de las dimensiones que se proponen posee mayor importancia, todas son necesarias, pero en ocasiones se utilizan los términos de capacidad y competencia al mismo nivel, sin llegar a distinguir su diferencia.

Según Gayol et al. (2008), la competencia investigativa está integrada por "capacidades cognoscitivas, tales como: manejar críticamente la bibliografía, seleccionar y delimitar el problema a investigar; abordar el trabajo tanto individual como grupalmente; formular hipótesis; precisar marco teórico, hipótesis y tesis; diseñar el proceso de la validación, verificación o legitimación de las hipótesis; estructurar y concretar un proyecto científico ..." (p. 4). Relacionar las competencias con capacidades traería consigo utilizar 
doi: http://dx.doi.org/10.15359/ree.18-2.9

URL: http://www.una.ac.cr/educare

CORREO:educare@una.cr

dos concepto diferentes, ya que la capacidad es entendida, según Rubinstein (1986, p. 78) como "propiedades o cualidades del hombre que lo hacen apto para realizar con éxito algunos tipos de actividad socialmente útiles"; mientras que Leontiev afirma que son "propiedades del individuo cuyo conjunto condiciona el éxito en el cumplimiento de determinada actividad" (Leontiev, 1986, p. 57). La capacidad es una potencialidad que puede convertirse en real o no; mientas que las competencias están dirigidas a una actuación por parte del individuo que le permita desempeñarse eficientemente: es una potencialidad manifestada que se evidencia en el desempeño.

Una de las limitaciones que posee este grupo es no tener en cuenta los valores asociados a la investigación científica, como la honestidad y la responsabilidad de la persona investigadora. Es válido aclarar que no afirmo que existen valores científicos, sino que estos se manifiestan de una manera específica en el transcurso de la realización de una investigación, por ejemplo, el no plagiar una información dada por otro autor o autora, la no falsificación de la información obtenida en la investigación, y la responsabilidad que posee la persona que investiga ante la institución que representa y la comunidad científica internacional.

Otra limitación es que no tiene en cuenta, en sus dimensiones, la motivación. Esta es la que representa los procesos que garantizan la toma de conciencia (reflexión metacognitiva) y el control (regulación metacognitiva) del proceso de construcción del conocimiento científico acerca de la realidad en dependencia, claro está, del fenómeno que se esté investigando.

Tampoco tiene en cuenta la experiencia social que posee el individuo, que a su vez puede contribuir a la eficiencia en cuanto al desarrollo de una investigación en menor tiempo. Esta experiencia se refiere a las particularidades del individuo que constituyen su arsenal histórico y que en un momento determinado pueden favorecer o limitar la actividad investigativa, por ejemplo: las condiciones tecnológicas, el acceso a información científica y el acceso directo del fenómeno a analizar.

Por último, no se tiene en cuenta una dimensión profesional, donde el sujeto desarrolle habilidades inherentes a su profesión y que haga a esta profesión distinta de las demás; por ejemplo, los graduados de ingeniería informática, según Shaw (2002), deben saber aplicar varios métodos investigativos de la informática: la simulación, el diseño, evaluación o análisis de una instancia particular y el método de desarrollo.

Las limitaciones antes mencionadas no permiten que la formación de la competencia investigativa sea del todo efectiva, al no tener en cuenta otros aspectos de la personalidad del sujeto como la motivación, la experiencia social y la dimensión profesional específica de la carrera universitaria; además, no se identifica la utilización de la interdisciplinariedad en las dimensiones que proponen los autores. 
doi: http://dx.doi.org/10.15359/ree.18-2.9

URL: http://www.una.ac.cr/educare

CORREO: educare@una.cr

\section{Fortalezas y debilidades del grupo 2}

Este grupo abordado es similar al anterior, ya que asume que la competencia investigativa es una integración de dimensiones cognitivas (conocimientos, habilidades), cualidades personales (actitudes, destrezas) y metacognitivas que permiten al estudiantado desempeñarse correctamente en una actividad investigativa; pero, además, agregan otra dimensión: la profesional.

Una fortaleza de este grupo consiste en que al abordar la dimensión profesional, las acciones y estrategias que puede realizar el profesor o profesora en el aula son más integradoras, pues relacionan esta competencia con el ámbito profesional de la carrera universitaria. Además, asumen todas las fortalezas del grupo 1.

En este grupo no se identifica el uso de la tecnología (solo se declara el uso de gestores bibliográficos y para el análisis de los datos recogidos en la investigación, no se aborda el uso de las tecnologías como apoyo en la realización de entrevistas y encuestas online, ni su análisis estadístico) ni la influencia de las condiciones tecnológicas; además, no se evidencia la inclusión de una dimensión de motivación ni de experiencia social, explicadas anteriormente.

También en este grupo, desde lo psicológico, se evidencia la utilización de los términos de competencia y capacidad, como iguales. Por ejemplo, Castillo (2011, p. 10) asume que la competencia cognitiva es en sí una "capacidad para la estructuración y dominio propio de cada uno de los procesos cognoscitivos e intelectuales que lleva a la construcción de habilidades de pensamiento y a su vez a diversas alternativas investigativas"; cuando en realidad desde lo psicológico, una dimensión cognitiva contiene los procesos y propiedades intelectuales, la base de conocimientos y el sistema de acciones generales y específicas.

Tomando en consideración a Fernández et al. (2003, El componente cognitivo, párr. 2): "los procesos y propiedades intelectuales hacen referencia al nivel de desarrollo alcanzado por el pensamiento y diferentes propiedades del funcionamiento intelectual que expresan la calidad procesal alcanzada por el sujeto cognoscente" (p. 7); por lo que se pueden determinar indicadores como la independencia, la originalidad y la fluidez.

La base de conocimiento presupone la apropiación, por parte de la persona profesional, de un amplio sistema de conocimientos estructurados significativamente, especializados y transferibles, como la política científica, los fundamentos filosóficos, sociológicos, psicológicos y axiológicos. Desde luego, esto depende del tipo de investigación y del tipo de profesión. Entre los posibles indicadores se encuentra la riqueza (cantidad y profundidad) de conocimientos sobre una o más áreas, en especial el nivel de conocimientos generales acerca de la investigación que posee el sujeto cognoscente; así como nivel de organización y sistematización de los conocimientos que posea.

Por último, el sistema de acciones generales y específicas, como afirman Fernández et al. (2003), es el dominio de determinadas habilidades, procedimientos, estrategias y capacidades que resultan indispensables para operar con la base de conocimientos y aplicar los saberes 
doi: http://dx.doi.org/10.15359/ree.18-2.9

URL: http://www.una.ac.cr/educare

CORREO: educare@una.cr

adquiridos en función de las diversas tareas inherentes a la construcción del conocimiento científico. Este se encuentra limitado a las etapas de la investigación científica o tecnológica, y las acciones que se deben de ejecutar en cada una de dichas etapas.

Si bien este grupo abarca otras dimensiones que el grupo 1 no consideró, todavía no permite el desarrollo de la competencia investigativa en su plenitud, ya que limita al profesorado universitario a tener en cuenta, en la dimensión cognitiva, solo los procesos cognitivos.

Desde lo pedagógico se hace hincapié en el desarrollo de habilidades comunicativas, observacionales, procedimentales, analíticas, tecnológicas e interpersonales; pero estas no se estructuran según las exigencias de las etapas de la investigación científica o tecnológica ni se integran con las características propias de la dimensión profesional específica asociada a la profesión del sujeto.

\section{Fortalezas y debilidades del grupo 3}

Este se encuentra fundamentado en el enfoque histórico-cultural de Vygotsky, defiende que la competencia investigativa es una configuración psicológica que permite la integración de componentes cognitivos, metacognitivos, motivacionales y cualidades de personalidad que se vinculan dialécticamente permitiéndole al sujeto desempeñarse correctamente en la actividad investigativa.

Esta concepción posee, como fortaleza, que atiende tanto el fundamento psicológico y pedagógico, como lo motivacional y cualidades de la personalidad. Se le brinda gran importancia al vínculo afectivo del sujeto hacia la actividad científica, que denotaría la presencia de las motivaciones intrínsecas; y a la satisfacción por la participación en esta actividad, lo que revelaría el cumplimiento de sus expectativas y la conservación de su autoestima. Además, se consideran indicadores como el compromiso con la solución de los problemas detectados, la independencia para manejar la información, la utilización correcta y completa del método científico, así como la actitud crítica y autocrítica para enjuiciar los puntos de vista propios y de otros especialistas.

En sus limitaciones se encuentra que no tiene en cuenta la experiencia social del sujeto ni la dimensión profesional. Además, se evidencia un papel preponderante de lo psicológico, cuando la persona humana es un ser bio-psico-social y su comportamiento está matizado por numerosos factores que se dan al unísono, y por los criterios imperantes de un determinado contexto. Además, la competencia solo se expresa una vez realizada la acción en un desempeño profesional determinado, pues en dependencia del tipo de profesión, la competencia investigativa se expresa de forma diferente, pues si bien esta es estándar en el sentido de las etapas de la investigación tecnológica (no todas las investigaciones conllevan a una tecnológica) o científica (básica o aplicada), la dimensión profesional le otorga particularidades que anteriormente se explicaron. Además, tampoco se hace alusión al uso de las tecnologías como soporte y ayuda al investigador o investigadora. 
doi: http://dx.doi.org/10.15359/ree.18-2.9

URL: http://www.una.ac.cr/educare

CORREO: educare@una.cr

\section{Fortalezas y debilidades del grupo 4}

De este grupo, si bien concebido en tesis doctorales en pedagogía, no se encuentran seguidores en la comunidad internacional, por lo menos en la revisión bibliografica realizada desde 2005-2013.

Los autores de este grupo asumen que la competencia investigativa es un sistema que resulta de la movilización los recursos cognitivos, metacognitivos, motivacionales; los valores éticos-profesionales, y la experiencia social propia de un sujeto. Este sistema permite un desempeño eficiente en situaciones investigativas vinculadas a su contexto laboral, donde la experiencia social propia del individuo es integrada a las demás dimensiones. Todas los sistemas se articulan de manera armónica en la actuación del sujeto con un enfoque configuracional.

Este sistema posee, en su estructura, tres dimensiones: la cognitiva, la afectiva-motivacional y la experiencia socio-individual, estrechamente articuladas en la actuación del sujeto.

Esta concepción también se encuentra fundamentada en el enfoque histórico-cultural de Vygostky y posee como fortaleza el reconocimiento de la persona humana como un ser bio-psico-social. Por lo que en el ámbito pedagógico hace referencia a la necesidad de tener en cuenta no solo lo cognitivo, sino también lo afectivo, destacándose la relación cognitivoafectivo en el proceso de enseñanza-aprendizaje y a partir de aquí la necesidad de la actividad, la comunicación, la internalización y la mediación, así como el papel de la educación en la conducción del desarrollo de la competencia investigativa.

Se destaca la actividad y su célula fundamental, la tarea, como medio para el desarrollo de competencias y expresión de la relación teoría y práctica. El vínculo entre teoría y práctica resulta imprescindible para la búsqueda de soluciones a la práctica educativa, desde su interpretación científica para su transformación.

Este grupo posee como limitación, el no reconocimiento de una dimensión profesional que propicie el desarrollo de habilidades profesionales específicas de la profesión. Además, considerar la experiencia social del sujeto como lo principal es una concepción muy atrevida, al asumir que es la experiencia social adquirida por el sujeto la que lo dirige a un desempeño exitoso. Ahora bien, si un individuo va a realizar por primera vez una investigación acorde al pregrado, ¿podrá la experiencia social del sujeto ayudarle en este sentido, si hasta el momento solo había realizado informes investigativos de asignaturas, e informes de clases tipo seminario y tipo taller? ¿Si el sujeto solo cursó en su carrera universitaria un curso (asignatura) de metodología de la investigación científica, podrá desempeñarse eficientemente en la actividad investigativa de la realización de una tesis? En mi opinión no; es a partir de la dimensión profesional que individuo puede ir desempeñándose como investigador a través de su interactuar en la práctica profesional

Es correcto asumir que para la realización de una investigación siempre el individuo parte de su experiencia, de sus conocimientos y de los problemas y necesidades que detectó y diagnosticó en la realidad profesional en que se desenvuelve; pero las 
doi: http://dx.doi.org/10.15359/ree.18-2.9

URL: http://www.una.ac.cr/educare

CORREO: educare@una.cr

habilidades asociadas a la investigación científicas no son las únicas que le ayudarán a resolver el problema de la investigación, sino también las habilidades profesionales y los conocimientos asociados a su profesión.

Además, la complejidad del mundo actual ha hecho que las investigaciones se realicen en grupo multidisciplinarios, integrando varias disciplinas y ciencias, y donde no siempre el grupo de investigación se encuentra en un mismo local de trabajo, ya que pueden ubicarse en lugares distantes, donde el uso de la tecnología es primordial para la investigación a distancia. Para ello hace falta desarrollar, en los futuros profesionales, no solo habilidades asociadas a la metodología de la investigación científica, sino también habilidades profesionales, tecnológicas y de trabajo grupal para la labor en comunidades científicas y de desarrollo, además de tener en cuenta la asimilación de conocimientos interdisciplinarios.

\section{Fortalezas y debilidades del grupo 5}

En este grupo se parte del enfoque complejo. Según Tobón (2008, citado en Tobón, 2008b, p. 5) se concibe el témino de competencia como:

Procesos complejos de desempeño con idoneidad en determinados contextos, integrando diferentes saberes (saber ser, saber hacer, saber conocer y saber convivir), para realizar actividades y/o resolver problemas con sentido de reto, motivación, flexibilidad, creatividad, comprensión y emprendimiento, dentro de una perspectiva de procesamiento metacognitivo, mejoramiento continuo y compromiso ético, con la meta de contribuir al desarrollo personal, la construcción y afianzamiento del tejido social, la búsqueda continua del desarrollo económicoempresarial sostenible, y el cuidado y protección del ambiente y de las especies vivas.

En esta cita se destacan seis aspectos fundamentales: procesos, complejidad, desempeño, idoneidad, metacognición y ética. Se asume que la competencia investigativa es un proceso complejo en el que se encuentran los componentes cognitivos, metacognitivos; cualidades de la personalidad; motivación; experiencia social y profesional del sujeto. Se concibe que el metacognitivo es el principal componente que integra a los demás. Claro está, el asumir el enfoque complejo se parte de la premisa de que tanto las partes del todo, como el todo mismo, es igual. Las competencias son procesos complejos porque implican la articulación y aplicación en tejido de diversos saberes y dimensiones humanas.

En el enfoque complejo, según Tobón (2008a):

Las competencias son un enfoque para la educación y no un modelo pedagógico, pues no pretenden ser una representación ideal de todo el proceso educativo, determinando cómo debe ser el tipo de persona a formar, el proceso instructivo, el proceso desarrollador, la concepción curricular, la concepción didáctica, la concepción epistemológica y el tipo de estrategias didácticas a implementar. Al contrario, las competencias son un enfoque 
doi: http://dx.doi.org/10.15359/ree.18-2.9

URL: http://www.una.ac.cr/educare

CORREO: educare@una.cr

porque sólo se focalizan en unos determinados aspectos conceptuales y metodológicos de la educación y la gestión del talento humano, como por ejemplo los siguientes: 1) integración de saberes en el desempeño, como el saber ser, el saber hacer, el saber conocer y el saber convivir; 2) la construcción de los programas de formación acorde con la filosofía institucional y los requerimientos disciplinares, investigativos, laborales, profesionales, sociales y ambientales; 3 ) la orientación de la educación por medio de criterios de calidad en todos sus procesos; 4) el énfasis en la metacognición en la didáctica y la evaluación de las competencias; y 5) el empleo de estrategias e instrumentos de evaluación de las competencias mediante la articulación de lo cualitativo con lo cuantitativo. (pp. 7-8)

Lo expuesto por Tobón (2008a) permite un acercamiento al cómo pudiera aplicarse este enfoque en la educación; pero, al no ser un modelo pedagógico, se carece de instrumentos, métodos, medios y herramientas con las cuales la didáctica pudiera dirigir el proceso de enseñanza-aprendizaje según el enfoque complejo. Asumir que este enfoque pueda aplicarse desde el punto de vista conceptual y metodológico de forma directa dentro del proceso didáctico es difícil, pues los fundamentos filosóficos, sociológicos, psicológicos y pedagógicos son distintos. El mismo autor refleja en sus escritos que existen los enfoques conductistas, funcionalistas, constructivista y el complejo, y que cada cual asume la formación y el desarrollo de competencias de forma distinta, por lo que en mi consideración se contradice, ya que si se conciben de formas distintas, ¿cómo se pueden integrar partiendo de fundamentos teóricos, conceptuales y metodológicos diferentes?

Este enfoque complejo es fundamental para comprender la formación compleja de los fenómenos sociales, y no solo estos, sino también el proceso de la personalidad del individuo. Permite un acercamiento a cómo formar competencias teniendo en cuenta tanto la complejidad del proceso de enseñanza-aprendizaje como la formación del individuo.

En este enfoque tiene como limitación que no reconoce la importancia de las tecnologías en la formación y desarrollo de la competencia investigativa y en el desempeño profesional del sujeto de forma integral, ya que estos autores proponen ciclos propedéuticos en el diseño curricular (ciclo propedéutico de técnico profesional, ciclo propedéutico de tecnología; y el ciclo profesional terminal de pregrado), pero no identifican la manera en que cada ciclo prepara al estudiante de forma integrada, ya que son concebidos por separados y solo existe un proyecto integrador que abarca los tres ciclos, donde, por supuesto, a medida que el estudiante va cursando por los diferentes ciclos irá desarrollado ese proyecto integrador.

\section{Conclusiones}

La sistematización teórica realizada permitió constatar cuales son las principales concepciones acerca del concepto de competencia investigativa y los componentes y dimensiones a tener en cuenta para su desarrollo. Se pudo determinar que las principales regularidades, según los autores consultados, son: 
doi: http://dx.doi.org/10.15359/ree.18-2.9

URL: http://www.una.ac.cr/educare

CORREO: educare@una.cr

1) La competencia es una integración de varios componentes como el cognitivo, el metacognitivo, la motivación y las cualidades personales que permiten el desempeño eficiente en la actividad investigativa.

2) Se debe tener en cuenta la relación académico-investigativo y laboral-investigativa.

3) Se encuentra relacionada con las etapas de la investigación científica o tecnológica, identificándose habilidades específicas por cada etapa.

4) Se debe considerar el trabajo en equipo, las relaciones interpersonales y la interdisciplinariedad.

Se pudo constatar en el análisis documental que si bien todos los autores consideran que la competencia investigativa se refleja en el desempeño del sujeto en la actividad investigativa en una profesión determinada, no se declara cómo, a partir de la actividad laboral propia de una profesión, se puede contribuir al desarrollo de dicha competencia a partir del uso del método científico e investigativo.

Se declara por los autores consultados la necesidad del uso de las tecnologías en el proceso investigativo; pero no se aclara cómo estas influyen en la calidad de la investigación, ya que solo lo reflejan en el uso de gestores bibliográficos, el análisis estadístico de los resultados obtenidos y la presentación de los resultados, pero no se refleja cómo las tecnologías pueden contribuir a una investigación a distancia, donde un grupo de investigadores interaccionan mediante la tecnología y es mediante esta que se comunican. Por lo que el uso efectivo de ella garantizaría, en cierta medida, el logro de los objetivos propuestos.

Existen diversos enfoques para la formación y desarrollo de competencias y cada uno con fundamentos teóricos y metodológicos diferentes, así como fortalezas y limitaciones. Afirmar que una es mejor que otra no sería conveniente, ya que depende de la concepción del mundo que se posea; pero sí creo pertinente afirmar algunos aspectos que se deben tener en cuenta para futuros trabajos en la formación y desarrollo de competencia investigativa, como son:

1) Las características personales del individuo y la formación de su personalidad en sus principales componentes cognitivos, motivacionales, experiencia social propia, metacognitivos y cualidades personales.

2) Las habilidades profesionales generales y particulares que debe asimilar y dominar el sujeto acorde con su profesión.

3) Las habilidades con el uso de la tecnología tanto para la realización de la actividad investigativa en todas sus etapas, como en el uso efectivo de esta de manera que le permita comunicarse e interactuar con otros investigadores en la realización de investigaciones a distancia en grupos multidisciplinarios.

4) La formación científica e investigativa interdisciplinaria y transdisciplinaria. 
doi: http://dx.doi.org/10.15359/ree.18-2.9

URL: http://www.una.ac.cr/educare

CORREO: educare@una.cr

5) La relación cognitiva-afectiva del proceso de enseñanza-aprendizaje.

6) La formación multilateral y armónica del individuo.

7) Potenciar el desarrollo de la competencia investigativa desde la actividad laboral propia del individuo en su profesión y no limitarla solamente a la actividad investigativa.

\section{Referencias}

Arbeláez, R., Hernández, N., O. y Pérez, M. I. (2006). El desarrollo de la competencia investigadora en los estudiantes de pregrado. Docencia Universitaria, 7(1), 5-10.

Association pour l'emploi des cadres (France) y Deloitte (France). (2010). Skills and competencies needed in the research field objectives 2020 [Habilidades y competencias necesarias en los objetivos de la actividad investigativa para el 2020]. París: APEC.

Balbo, J. (2008). Formación en competencias investigativas, un nuevo reto en las universidades. Caracas: Universidad Central de Venezuela.

Baltrūna, A. (invierno, 2013). The Essence of Learning Methodological Model and its Application in Developing Tourism Students' Research Competence [La esencia del aprendizaje del modelo metodológico y su aplicación en el desarrollo de competencia investigativa en los estudiantes de turismo]. The Macrotheme Review, 2(1), 19-25. Recuperado de http:// macrotheme.com/yahoo site admin/assets/docs/BaltrunaMR21f.345104252.pdf

Barnett, B. y Muth, R. (mayo, 2008). Using Action-Research Strategies and Cohort Structures to Ensure Research Competence for Practitioner-Scholar Leaders [El uso de la estrategia investigación-acción y la estructura de cohortes para garantizar la competencia investigativa en lideres profesionales]. Journal of Research on Leadership Education, 3(1), 1-42. doi:10.1177/194277510800300101

Barrón, C. (2000). La educación basada en competencias en el marco de los procesos de globalización, en M. A. Valle (Coord.), Formación en competencias y certificación profesional (pp. 17-44). México: Universidad Nacional Autónoma de México.

Bezrukova, N., y Bezrukov, A. (2013). Methodological basis of Development of Research Competence of Secondary School Students in the Network Research Community [Bases metodológicas para el desarrollo de la competencia investigativa en estudiantes de secundarias desde las comunidades de investigadores]. $C B U$ international conference on integration and innovation in science and education, 144-151.

Bolgzda, I. y Olehnovica, E. (2012). Structure of Doctoral Students' Research Competences in Sustainability Context [Estructura de la competencia investigativa en estudiantes de doctorado en contexto de sostenibilidad]. World Academy of Science, Engineering and Technology, 66, 412-415. Recuperado de http://waset.org/Publications/structure-ofdoctoral-students-research-competences-in-sustainability-context/4183 
doi: http://dx.doi.org/10.15359/ree.18-2.9

URL: http://www.una.ac.cr/educare

CORREO: educare@una.cr

Burke, L.E., Schlenk, E. A., Sereika, S. M., Cohen, S. M., Happ, M. B., y Dorman, J. S. (noviembrediciembre, 2005). Developing Research Competence to Support Evidence-Based Practice [La práctica basada en evidencia como apoyo para el desarrollo de la competencia investigativa]. Journal of Professional Nursing, 21(6), 358-363.

Castillo, S. (julio-diciembre, 2008). Competencias investigativas desarrolladas por docentes de matemática. Acta Scientia. Revista de Ensino de Ciências e Matemática, 10(2), 3-7.

Castillo, S. (junio, 2011). Evaluación de competencias investigativas. Trabajo presentado en la XIII Conferencia Interamericana de Educación Matemática (CIAEM). Recife, Brasil: Recuperado de http://www.cimm.ucr.ac.cr/ocs/files/conferences/1/schedConfs/1/papers/1588/ submission/review/1588-4051-1-RV.pdf

Cejas, E. (2006). La formación por competencias laborales: Proyecto de diseño curricular para el técnico en farmacia (Tesis doctoral). Universidad de Ciencias Pedagógicas Enrique José Varona., La Habana, Cuba.

Chu, S., Chow, K., Tse, S.-K., y Collier, C. (enero, 2008). Grade 4 Students' Development of Research Skills Through Inquiry-Based Learning Projects [Desarrollo de habilidades investigativas en estudiantes de cuarto grado a través de proyectos basados en indagación]. School Libraries Worldwide, 14(1), 10-37. Recuperado de http://www.iasl-online.org/pubs/slw/ jan08-chu.htm

Colectivo de Autores. (2003). Metodología de la investigación educacional. Retos y polémicas actuales. La Habana: Editorial Félix Varela.

Connor, P. E. (julio, 1972). Scientific Research Competence. Two Forms of Collegial Judgment [Competencias investigativas científicas. Dos formas de desarrollar]. The Pacific Sociological Review, 15(3), 355-366.

Contreras, Z. (2010). Las competencias investigativas. Educando. Ministerio de Educación de la República Dominicana. Recuperado de http://www.educando.edu.do/ files/1713/3190/5240/Las Competencias Investigativas1.pdf

Delamare Le Deist, F., y Winterton, J. (marzo, 2005). What Is Competence? [¿Qué se entiende por competencia?]. Human Resource Development International, 8(1), 27-46. Recuperado de http://www.slideshare.net/bradpenn/what-is-competence

Fernández, A. M., Llivina, M. J., Arencibia, V., Hernández, R., y Castellanos, B. (2003). Informe técnico del resultado: Esquema conceptual, referencial y operativo (ECRO) sobre la investigación educativa. La Habana: Universidad de Ciencias Pedagógicas José Enrique Varona. Recuperado de http://www.monografias.com/trabajos76/desarrollo-competenciainvestigacion-profesionales-educacion/desarrollo-competencia-investigacionprofesionales-educacion2.shtml 
doi: http://dx.doi.org/10.15359/ree.18-2.9

URL: http://www.una.ac.cr/educare

CORREO: educare@una.cr

Gallardo, O. (julio-diciembre, 2003). Modelo de formación por competencia para investigadores. Contexto e Educação, 18(70), 9-25. Recuperado de https://www.revistas.unijui.edu.br/ index.php/contextoeducacao/article/view/1141/895

Gayol, M., Montenegro, S. M., Tarrés, M. C., y D’Ottavio, A. E. (2008). Competencias investigativas. Su desarrollo en carreras del área de la salud. UNI-PLURI/VERSIDAD, 8(2), 1-8. Recuperado de http://aprendeenlinea.udea.edu.co/revistas/index.php/unip/article/viewFile/950/823

Glass, R. L., Vessey, I., y Ramesh, V. (Junio, 2002). Research in Software Engineering: An Analysis of the Literature [La investigación en la ingeniería del software: Un análisis desde la bibliografía]. Information and software Technology, 44(8), 491-506.

Gómez, D. E. (2009). Estrategia pedagógica para el desarrollo de la competencia investigativa en la formación del bachiller técnico en alimentos (Tesis doctoral). Universidad de Ciencias Pedagógicas Héctor Zaldívar Pineda, La Haba, Cuba.

Gray, C. (2007). Research Competencies Framework [Entorno de trabajo de la competencia investigativa]. England: Faculty of General Dental Practice (UK). The Royal College of Surgeons of England. Recuperado de http://www.fgdp.org.uk/ assets/pdf/research/ research\%20competencies.pdf

Hakkarainen, K., Lipponen, L., Jarvela, S., y Niemivirta, M. (1999). The Interaction of Motivational Orientation and Knowledge-Seeking Inquiry in Computer-Supported Collaborative Learning [La interacción de la orientación motivacional y la búsqueda del conocimiento en el aprendizaje colaborativo y apoyado por las tecnologías]. Journal of Educational Computing Research, 21(3), 263-281.

Irigoin, M., y Vargas, F. (2002). Módulo 2. La formación basada en competencias. Unidad 5. El diseño de la formación basada en competencias (FBC). En M. Irigoin. y F. Vargas (Auts.), Competencia laboral: Manual de conceptos, métodos y aplicaciones en el sector saud (pp. 175-214). Montevideo: CINTERFOR-OPS.

Lee, H.-F., Miozzoa, M., y Laredob, P. P. (setiembre, 2010). Career Patterns and Competences of PhDs in Science and Engineering in the Knowledge Economy: The Case of Graduates from a UK Research-Based University [Patrones y competencias de los estudiantes de doctorados de las carreras de ciencia en la economía del conocimiento e ingeniería: El caso de los graduados en una universidad de Reino Unido]. Research Policy, 39(7), 869-878.

Leontiev, A. N. (1986). Sobre la formación de las capacidades. En En I. I. Iliasov y V. Ya. Liaudis (Comps.), Antología de la psicología pedagógica y de las edades (pp. 57-67). La Habana: Editorial Pueblo y Educación.

Maldonado, L. F., Landazábal, D. P., Hernández, J. C., Ruíz, Y., Claro, A., Vanegas, H. y Cruz, S. (abril-junio, 2007). Visibilidad y formación en investigación. Estrategias para el desarrollo de competencias investigativas. Studiositas, 2(2), 43-56. Recuperado de http://dialnet. unirioja.es/servlet/articulo?codigo $=2719652$ 
doi: http://dx.doi.org/10.15359/ree.18-2.9

URL: http://www.una.ac.cr/educare

CORREO: educare@una.cr

Morin, E. (2002). Los siete saberes necesarios para la educación del futuro. Buenos Aires: Nueva Visión.

Muñoz, J. F., Quintero, J., y Munévar, R. A. (2006). Cómo desarrollar competencias investigativas en educación. Bogotá: Magisterio.

Ortega, E., y Jaik, A. (julio, 2010). Escala de evaluación de competencias investigativas. Revista Electrónica Praxis Investigativa ReDIE, 2(3), 72-80.

Pérez, N. E., y Setién, E. (octubre, 2008). La interdisciplinariedad y la transdisciplinariedad en las ciencias: Una mirada a la teoría bibliológico-informativa. Acimed, 18(4). Recuperado de http://scielo.sld.cu/pdf/aci/v18n4/aci31008.pdf

Pla, R. (marzo, 2005). Las competencias profesionales para el desempeño del docente en la educación de los alumnos desde un enfoque integrador. Taller de Pedagogía. Taller llevado a cabo en el Congreso Internacional Pedagogía. La Habana, Cuba.

Proyecto Tuning-América Latina. (2007). Reflexiones y perspectivas de la educación superior en América Latina (Informe final -proyecto Tuning- América Latina: 2004-2007). Bilbao: Universidad de Deusto.

Quigley, B. A. y Kuhne, G. W. (Spring, 1997). Creating Practical Knowledge Through Action Research: Posing Problems, Solving Problems, and Improving Daily Practice [Creación de conocimiento a través de la investigación-acción: Presentación, problemas, solución de problemas y la mejora de la práctica diaria]. New Directions for Adult and Continuing Education, 73.

Rodríguez, M. (2000). Formación reflexiva-creativa de competencias investigativas en los docentes. Buenos Aires: CLACSO.

Rubinstein, S. L. (1986). El problema de las capacidades y las cuestiones relativas a la teoría psicológica. Compilado por I. I. Iliasov y V. Ya. Liaudis, Antología de la psicología pedagógica y de las edades (pp. 60-97). La Habana: Editorial Pueblo y Educación.

Sánchez, P. y Tejeda, R. (2010). El proceso de formación investigativa del profesional ingeniero y la(s) competencia(s) investigativa(s). Pedagogía Universitaria, 15(4), 37-47. Recuperado de http://cvi.mes.edu.cu/peduniv/index.php/peduniv/article/view/547/544

Santos, J. (2005). Modelo pedagógico para el mejoramiento del desempeño pedagógico profesional de los profesores de agronomía de los Institutos Politécnicos Agropecuarios (Tesis doctoral). Instituto Superior Pedagógico Enrique José Varona, La Habana, Cuba.

Shaw, M. (2002). What Makes Good Research in Software Engineering? [¿Cómo hacer una correcta investigación en Ingeniería de software?]. International Journal of Software Tools for Technology Transfer, 4, 1-7.

Tobón, S. (2008a). Gestión curricular y ciclos propedéuticos. Bogotá: ECOE. 
doi: http://dx.doi.org/10.15359/ree.18-2.9

URL: http://www.una.ac.cr/educare

CORREO: educare@una.cr

Tobón, S. (2008b). La formación basada en competencias en la educación superior. El enfoque complejo. Bogotá: Instituto Cife. Tobón, S., Rial, A., Ángel, M. y García, J. A. (2006). Competencias, calidad y educación superior. Bogotá: Magisterio.

Tobón, S., Rial, A., Ángel, M. y García, J. A. (2006). Competencias, calidad y educación superior. Bogotá: Magisterio. Velásquez, R. (2007). De esto y aquello sobre la competencia investigativa. Avances Investigación en Ingenierías, 7, 5-11. http://www.revistaavances.co/ objects/docs/Avances 7/a7 art1 competencia inv.pdf

Vygotsky, L. S. (1982). Pensamiento y lenguaje. La Habana: Pueblo y Educación.

(6) Cómo citar este artículo en APA:

Estrada, O. (mayo-agosto, 2014). Sistematización teórica sobre la competencia investigativa. Revista Electrónica Educare, 18(2), 177-194.

doi: http://dx.doi.org/10.15359/ree.18-2.9

Nota: Para citar este artículo en otros sistemas puede consultar el hipervínculo "Como citar el artículo" en la barra derecha de nuestro sitio web:

http://www.revistas.una.ac.cr/index.php/EDUCARE/index 